\title{
Relation between the serum albumin level and nutrition supply in patients with pressure ulcers : retrospective study in an acute care setting
}

\author{
Hirotaka Sugino', Ichiro Hashimoto', Yuka Tanaka², Soshi Ishida', Yoshiro Abe', \\ and Hideki Nakanishi ${ }^{1}$ \\ ${ }^{1}$ Department of Plastic and Reconstructive Surgery, Institute of Health Biosciences, the University of \\ Tokushima Graduate School, Tokushima, Japan, ${ }^{2}$ Department of Nutrition and Metabolism, Institute \\ of Health Biosciences, the University of Tokushima Graduate School, Tokushima, Japan
}

\begin{abstract}
This retrospective study examined the validity of the commonly used serum albumin level as an indicator of nutrition status of patients with pressure ulcer(s), particularly because the serum albumin level is affected by various factors and may not be specific to malnutrition. Specifically, we investigated whether nutrition supply or inflammation affects the serum albumin level in 82 patients with pressure ulcers(s) (29 in whom pressure ulcer was present upon admission and 53 in whom pressure ulcer developed after hospital admission). Serum albumin levels, blood test including C-reactive protein (CRP) levels and blood count, caloric intake, and depth and healing of pressure ulcers were compared between various subgroups of patients. Serum albumin levels correlated with red blood cell counts and hemoglobin and CRP levels but not with caloric intake. The correlation with CRP before and after several weeks of pressure ulcer treatment was negative. The serum albumin level upon admission was higher in patients in whom the ulcer healed than in those in whom it did not heal as well as in patients who were discharged than in those who died in the hospital. The serum albumin level appears to reflect inflammation, wound healing, and disease severity rather than nutrition supply in patients with pressure ulcer. J. Med. Invest. 61 : 15-21, February, 2014
\end{abstract}

Keywords : serum albumin level, nutrition status, pressure ulcer, C-reactive protein, inflammation

\section{INTRODUCTION}

Butterworth reported in 1974 that an alarming number of hospitalized patients were malnourished and hypoalbuminemic (1). Since then, it has been believed that the serum albumin level is one of the

Received for publication June 26, 2013 ; accepted July 24, 2013.

Address correspondence and reprint requests to Ichiro Hashimoto, MD, PhD, Department of Plastic and Reconstructive Surgery, the University of Tokushima Graduate School, Kuramoto-cho, Tokushima 770-8503, Japan and Fax : +81-88633-7297. best indicators of nutrition status and that the poor nutrition status marked by hypoalbuminemia means severe protein malnutrition in particular (2-8). In the 1990 s, some investigators questioned whether the serum albumin level is a reliable indicator of nutrition status. As a case in point, anorexia nervosa patients are malnourished but have a normal serum albumin level (9). In addition, some patients after severe trauma or surgery show severe hypoalbuminemia but are not malnourished. Furthermore, even patients with increasing body weight under adequate nutritional support can show low serum albumin 
levels (10-16). Nevertheless, the serum albumin level has been used in subsequent risk assessments for pressure ulcer development and has been considered a marker for pressure ulcer healing (17-19).

We conducted a retrospective study in an acute care setting to clarify the significance of the serum albumin level in assessing the nutrition supply of patients with pressure ulcer(s) by analyzing serum albumin levels with respect to caloric intake, blood test results, and survival outcomes and by comparing values between various patient subgroups.

\section{METHODS}

\section{Patients}

The study included 82 patients with pressure ulcer(s) who were treated at Tokushima University Hospital between April 2007 and December 2009. Only patients who underwent five or more blood tests between the time of admission and the time of discharge or in-hospital death were included in the study. No patient who underwent blood transfusion was included. In addition, no patient who was monitored for fewer than 4 weeks was included. Characteristics of the study patients, including the reasons for hospitalization, are shown on Table 1 . The pressure ulcers developed in 29 of the patients before admission and in 53 after admission.

\section{Data collection}

Patient data including demographic characteristics, blood test results, and caloric intake were collected from medical records. Blood test results included serum albumin levels, C-reactive protein (CRP) levels, white blood cell (WBC) count, red blood cell (RBC) count, and hemoglobin (Hb) levels.

Each patient's caloric intake was calculated according to the amount of enteral or parenteral nutrition, contents of the parenteral or enteral solution, and feeding rate, from the patient's medical record. Caloric intake from the day of a blood test until the day of the next blood test was totaled and then averaged per number of days between tests to yield a daily value. In addition, basal energy expenditure (BEE, as determined by the Harris-Benedict Equation), caloric intake/BEE, and caloric intake/body weight were calculated.
Table 1. Patient characteristics $(n=82)$

\begin{tabular}{ll}
\hline Variable & Value \\
\hline Age & $64.4 \pm 15.1$ years \\
Sex & \\
$\quad$ Male & $50(61 \%)$ \\
$\quad$ Female & $32(39 \%)$ \\
Number of in-hospital deaths & $28(34 \%)$ \\
Average period of hospitalization & $76.3 \pm 61.7$ days \\
Body weight & \\
$\quad$ Male & $63.5 \pm 13.6 \mathrm{~kg}$ \\
$\quad$ Female & $52.0 \pm 15.2 \mathrm{~kg}$ \\
Body mass index & $23.5 \pm 5.5$ \\
Condition responsible for the formation & \\
of pressure ulcers & \\
Surgery & $26(32 \%)$ \\
$\quad$ End-stage malignant disorder & $20(24 \%)$ \\
Neurogenic disorder & $19(23 \%)$ \\
Respiratory disorder & $5(6 \%)$ \\
Other & $12(15 \%)$ \\
Stage of pressure ulcers & \\
I & $11\left(5^{*}\right)$ \\
II & $52\left(41^{*}\right)$ \\
III & $2\left(1^{*}\right)$ \\
IV & $14\left(6^{*}\right)$ \\
\hline
\end{tabular}

Number and percentage of patients are shown unless otherwise indicated.

*Number of patients in whom pressure ulcer developed after admission.

Pressure ulcer stages : I, non-blanchable erythema; II, partial thickness skin loss ; III, full thickness skin loss ; IV, full thickness tissue loss (National Pressure Ulcer Advisory Panel's pressure ulcer staging system).

\section{Analyses}

Correlation between serum albumin levels and biochemical and clinical measurements

Serum albumin levels were analyzed in relation to six variables : CRP level, WBC count, RBC count, $\mathrm{Hb}$ level, caloric intake/BEE, and caloric intake/ body weight. In addition, CRP levels were analyzed in relation to WBC count. The average, median, and distribution of each correlation coefficient (Pearson's product-moment correlation coefficient) were calculated. Significance of the correlation was determined by Student's t-distribution.

\section{Serum albumin levels and caloric intake}

To analyze serum albumin levels in relation to caloric intake, the patients were divided into two groups : a greater caloric intake group (average caloric intake was over $25 \mathrm{kcal} / \mathrm{kg} /$ day during the 
4-to-8 week- pressure ulcer treatment period) and a lesser caloric intake group (average caloric intake was under $25 \mathrm{kcal} / \mathrm{kg} /$ day during this period). Serum albumin levels were obtained before pressure ulcer treatment and 4 to 8 weeks after treatment was started in each group. The two values were compared between the two different caloric intake groups by means of paired t-test.

Change in serum albumin levels in relation to CRP levels

To analyze serum albumin levels in relation to CRP levels, the patients were divided into three groups : those in whom serum albumin increased by more than $0.3 \mathrm{~g} / \mathrm{dL}$, those in whom serum albumin decreased by more than $0.3 \mathrm{~g} / \mathrm{dL}$, and those in whom serum albumin changed only minimally (between +0.3 and $-0.3 \mathrm{~g} / \mathrm{dL}$ ). The changes were based on the difference in CRP levels recorded before the pressure ulcer treatment and those recorded 4 to 8 weeks after the treatment was started in each group. Change in the CRP level in each group was analyzed by Wilcoxon signed-rank test.

Serum albumin levels and post-admission development of pressure ulcers

Serum albumin levels were analyzed in the 53 patients in whom pressure ulcers developed after admission. These patients were divided into two groups : those with shallow Stage I ulcers and those with deep Stage II to IV ulcers. The stages were assigned according to the National Pressure U1cer Advisory Panel's pressure ulcer staging system (http : //www.npuap.org/resources/educationaland-clinical-resources/). These patients were also divided into two groups based upon whether ulcers healed or did not heal during the period of hospitalization. Thus, the serum albumin levels upon admission were compared between patients with shallow ulcers and patients with deep ulcers and also between patients in whom the ulcers healed and those in whom they did not heal. Analysis was made by Mann-Whitney U test.

\section{Serum albumin levels and survival outcomes}

To analyze serum albumin levels in relation to survival outcomes, the patients were divided into two groups : those who died during their hospital stay and those who were discharged. Serum albumin levels upon admission and serum albumin levels after 4 to 8 weeks of treatment were compared within each group and analyzed by paired t-test. In addition, serum albumin levels were compared between patients who died during their hospital stay and those who were discharged and then analyzed by Mann-Whitney U test. Twenty-eight patients died during their hospital stay, but three of these patients were omitted from the survival analysis because serum albumin levels just before death were lacking.

\section{Statistical analyses}

Data are expressed as mean $( \pm$ standard deviation [SD]). All analyses were performed with Microsoft Excel 2007 (Microsoft Corporation, Redmond, WA, USA). Significance was accepted at $\mathrm{p} \leq 0.05$.

\section{RESULTS}

Correlation between serum albumin levels and biochemical and clinical measurements

Serum albumin levels correlated positively and significantly with $\mathrm{Hb}$ levels and $\mathrm{RBC}$ counts and negatively and significantly with CRP levels $(p<$ 0.01 ). Serum albumin levels did not correlate with WBC counts. However, significant positive correlation was found between CRP levels and WBC counts $(p<0.05)$. No correlation was found between serum albumin levels and caloric intake (Table 2).

\section{Serum albumin levels and amount of the caloric intake}

In greater caloric intake group, serum albumin levels before pressure ulcer treatment and after the start of pressure ulcer treatment were $2.8 \pm 0.5 \mathrm{(g} /$ $\mathrm{dL})$ and $2.6 \pm 0.8$, respectively. In lesser caloric intake group, serum albumin levels before pressure

Table 2. Correlation between serum albumin, CRP, and other variables

\begin{tabular}{lcc}
\hline & Albumin level & CRP level \\
\hline $\mathrm{CRP}(\mathrm{n}=82)$ & $-0.446 \pm 0.415^{\dagger \dagger}$ & \\
$\mathrm{WBC}(\mathrm{n}=82)$ & $-0.0354 \pm 0.529$ & $0.246 \pm 0.470^{*}$ \\
$\mathrm{Hb}(\mathrm{n}=82)$ & $0.551 \pm 0.424^{* *}$ & \\
$\mathrm{RBC}(\mathrm{n}=80)$ & $0.545 \pm 0.412^{* *}$ & \\
$\begin{array}{l}\text { Caloric intake/BEE } \\
\quad(\mathrm{n}=60)\end{array}$ & $0.0999 \pm 0.537$ & \\
$\begin{array}{l}\text { Caloric intake/body } \\
\quad \text { weight }(\mathrm{n}=59)\end{array}$ & $0.113 \pm 0.542$ & \\
\hline
\end{tabular}

Average and standard deviation of correlation coefficients are shown. $* \mathrm{p}<0.05$ (positive correlation) vs. CRP level; $* * \mathrm{p}<0.01$ (positive correlation) vs. albumin level $;{ }^{+\dagger} \mathrm{p}<0.01$ (negative correlation) vs. albumin level, by Student's t-distribution. 
ulcer treatment and after pressure ulcer treatment were $2.4 \pm 0.6(\mathrm{~g} / \mathrm{dL})$ and $2.4 \pm 0.8$, respectively (Fig. 1). No significant difference was found in the serum albumin levels obtained before and after the start of pressure ulcer treatment in either the greater or and lesser caloric intake group.

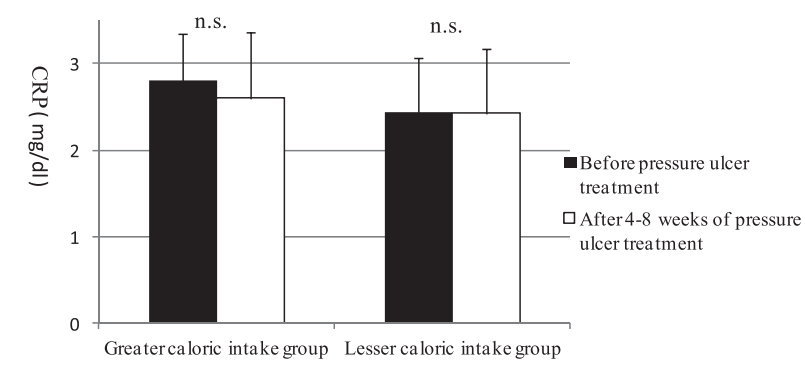

Figure 1. Serum albumin level per nutritional supply Greater caloric intake group $(\mathrm{n}=16) ;>25 \mathrm{kcal} / \mathrm{kg} /$ day, Lesser caloric intake group $(\mathrm{n}=16) ;<25 \mathrm{kcal} / \mathrm{kg} /$ day No significant within-group differences were found.

Change in serum albumin levels in relation to CRP levels

In the group of patients in whom serum albumin increased, the CRP level before pressure ulcer treatment and after the start of pressure ulcer treatment were $8.8 \pm 9.7(\mathrm{~g} / \mathrm{dL})$ and $1.7 \pm 1.9$, respectively. In the minimal change group, the CRP levels before pressure ulcer treatment and after the start of pressure ulcer treatment were $3.2 \pm 4.8(\mathrm{~g} / \mathrm{dL})$ and $3.0 \pm 3.8$, respectively. In the group in which serum albumin decreased, the CRP levels before pressure ulcer treatment and after the start of pressure ulcer treatment were $3.2 \pm 3.2(\mathrm{~g} / \mathrm{dL})$ and $7.5 \pm 6.8$, respectively. The CRP levels between before and after the treatment were significantly different $(p<0.01)$ in the albumin increase and decrease groups by Wilcoxon signed-rank test (Fig. 2).

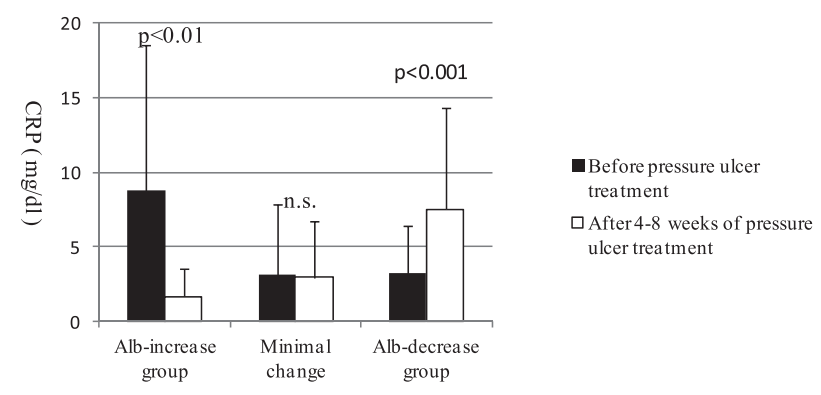

Figure 2. CRP levels per change in serum albumin Alb-increase group $(n=26)$; serum albumin increased by more than $0.3 \mathrm{~g} / \mathrm{dL}$, Minimal change group $(\mathrm{n}=19)$; serum albumin changed only minimally (between +0.3 and $-0.3 \mathrm{~g} / \mathrm{dL}$ ), Alb-decrease group $(n=16)$; serum albumin decreased by more than $0.3 \mathrm{~g} / \mathrm{dL}$.

\section{Serum albumin levels and development of pressure} ulcers

Among the 53 patients in whom pressure ulcers developed during their hospitalization, the admission serum albumin level for those in whom shallow ulcers developed was $3.1( \pm 0.6) \mathrm{g} / \mathrm{dL}$, and the admission level in those in whom deep ulcers developed was $3.1( \pm 0.8) \mathrm{g} / \mathrm{dL}$. The two albumin levels did not differ significantly. The admission serum albumin level of patients in whom the ulcers healed was $3.4( \pm 0.7) \mathrm{g} / \mathrm{dL}$, and the admission level of those in whom ulcers did not heal was $2.8( \pm 0.6) \mathrm{g} / \mathrm{dL}$. These two albumin levels did differ significantly $(\mathrm{p}<$ $0.01)$.

\section{Serum albumin levels and survival outcomes}

Among patients who were discharged, the serum albumin level upon admission $(3.2[ \pm 0.6] \mathrm{g} / \mathrm{dL})$ did not differ significantly from the level recorded near the time of discharge $(2.9[ \pm 0.6] \mathrm{g} / \mathrm{dL})$. Among patients who died in the hospital, the serum albumin level near the time of death $(1.9[ \pm 0.4] \mathrm{g} / \mathrm{dL})$ was significantly lower than the level at the time of admission $(2.6[ \pm 0.5] \mathrm{g} / \mathrm{dL})(\mathrm{p}<0.001)$. Serum albumin levels at the time of admission and after the 4 to 8 weeks of treatment were significantly higher in patients who survived than the respective levels in patients who died $(\mathrm{p}<0.001)$.

\section{Case Reports}

Case 1 : A 34-year-old woman was hospitalized under the diagnosis of putaminal hemorrhage. She underwent the operation to remove hematoma three times in her admission. Pressure ulcer of Stage IV developed at left auricle when she underwent the second surgical drainage of the brain abscess 18 days after admission. The pressure ulcer was not healed when she discharged 79 days after admission. Serum albumin levels correlated negatively with CRP levels (Fig. 3).

Case 2 : A 60-year-old man was hospitalized under diagnosis of interstitial pneumonia, diabetes mellitus and quadriplegia because of Guillain Barré syndrome. At the time of admission, he already had pressure ulcer (Stage IV) at his right heel. Drainage of retropharyngeal abscess was performed 23 and 31 days after admission. When he died 50 days after admission, the pressure ulcer (Stage III) still remained. Serum albumin levels correlated negatively with CRP levels. Both levels did not correlate with the condition of his pressure ulcer (Fig. 4). 


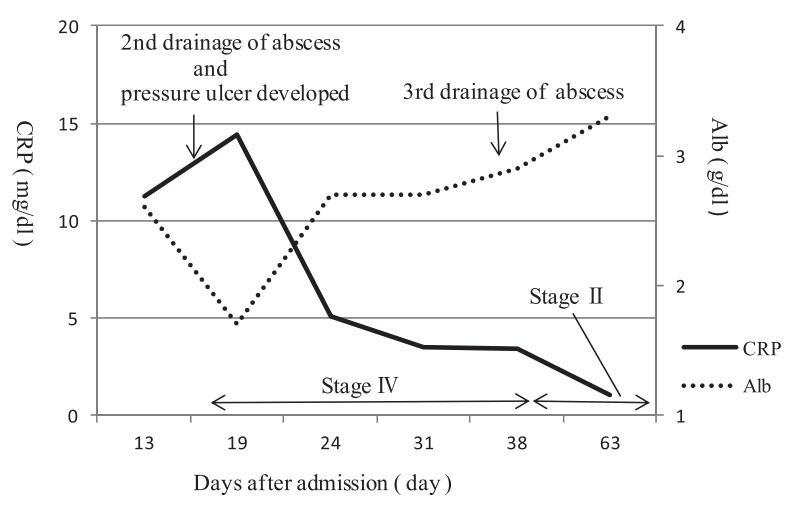

Figure 3. Time course of serum albumin and CRP levels and stage of the pressure ulcer in Case 1. Stage II, partial thickness skin loss ; Stage IV, full thickness tissue loss (National Pressure Ulcer Advisory Panel's pressure ulcer staging system).

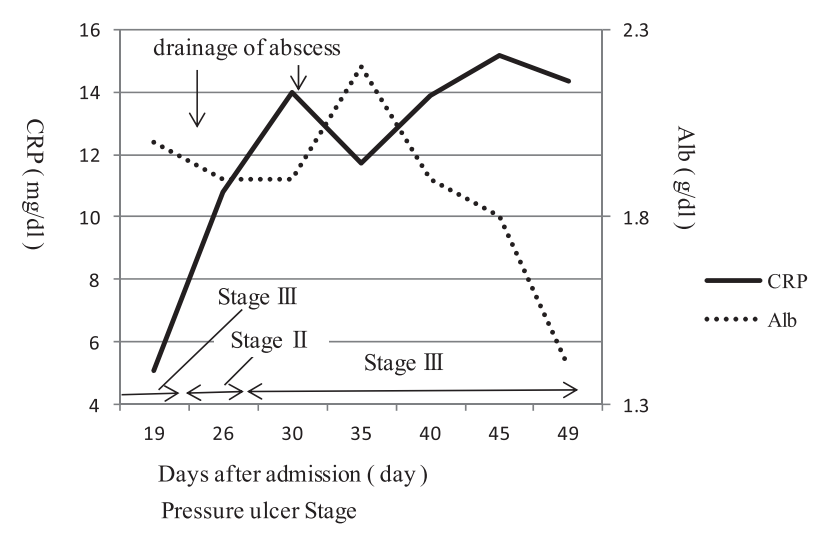

Figure 4. Time course of serum albumin and CRP levels and stage of the pressure ulcer in Case 2. Stage II, partial thickness skin loss ; Stage III, full thickness skin loss (National Pressure Ulcer Advisory Panel's pressure ulcer staging system).

\section{DISCUSSION}

It is still believed that the serum albumin level is a useful indicator of nutrition status and that malnutrition is characterized by a low serum albumin level. However, because the half-life of serum albumin is about 20 days and the body pool of serum albumin is large, the serum albumin level does not respond readily to the patient's nutrition status in the acute phase of severe illness or injury. Furthermore, the serum albumin level is affected by a variety of factors such as insulin, thyroid hormone, inflammatory cytokines, hypermetabolism, malabsorption, and intravascular volume overload (16). The decrease in serum albumin resulting from inflammatory cytokines is a most important issue among hospitalized patients (20). Inflammatory cytokines such as IL-1,
IL-6, and TNF- $\alpha$ increase synthesis of acute-phase proteins such as CRP to protect the body from trauma. At the same time, the inflammatory cytokines decrease synthesis of constitutive proteins such as serum albumin and increase its degradation. The inflammatory cytokines also promote capillary permeability and leakage of serum albumin into extravascular space. Thus, it is supposed that there is an inverse correlation between the CRP and serum albumin levels, although the difference in half-lives results in a time lag between the two biomarkers. The inflammation in our patients was presumed to have been caused not only by the pressure ulcers but also by the patients' disease states and surgeries ; all were acute care patients.

We found a significant positive correlation between the serum albumin level and the Hb level and $\mathrm{RBC}$ count, indicating that inflammation is related to the synthesis of $\mathrm{Hb}$. Hepcidin, an acutephase protein, is increased by IL- 6 in inflammatory disorders. The hepcidin decreases synthesis of $\mathrm{Hb}$ because it is a negative regulator of intestinal iron absorption and iron release from macrophages (21). In our study, an increase in the CRP level was related to a decrease in the serum albumin level, $\mathrm{Hb}$ level, and RBC count. This result suggests that the decrease in these factors is affected not by malnutrition but rather by inflammation. Therefore, the serum albumin level is not sufficient for assessment of nutrition supply.

The serum albumin level is still used to assess the adequacy of nutritional management in many institutions. However, it has been reported that nutritional support for malnourished patients has no direct effect on their serum albumin levels $(22,23)$. In agreement with results of previous studies, we found no correlation between the serum albumin level and caloric intake. Furthermore, there was no difference in serum albumin levels between our greater and lesser caloric intake groups during pressure ulcer treatment. We often encountered cases in which the serum albumin level did not increase despite nutritional support with adequate calories and protein. These findings suggest that the serum albumin level is not suitable for assessment of nutrition support.

The serum albumin level is also reported to be a useful indicator of disease prognosis, development of complications, and mortality (24-29). To investigate the relation between the serum albumin level and inflammation as an indicator of disease severity, we compared change in the CRP level between patients grouped according to whether their serum 
albumin level increased, decreased, or changed only minimally during their hospitalization. The CRP level decreased in the albumin-increase group and increased in the albumin-decrease group, suggesting that inflammation and the disease condition are improving when the serum albumin level is increasing and that the inflammation and disease condition are worsening when the serum albumin level is decreasing. Additionally, we found that the serum albumin level increased in the CRP-decrease group and decreased in the CRP-increase group (data not shown). Therefore, we believe that the serum albumin level can serve as at least one useful indicator of inflammation and disease status. We did not research serum proteins in addition to serum albumin. However, several groups have reported that other serum proteins being used to assess nutrition status, such as prealbumin and transferrin, are also affected by inflammation (30).

A low serum albumin level is reported to be a predictor of pressure ulcer development (17-19). To determine whether the serum albumin level is indeed a predictor of pressure ulcer or an indicator of pressure ulcer healing, we analyzed serum albumin levels in relation to depth of the pressure ulcer and healing of the pressure ulcer in the patients in whom the pressure ulcer developed after the admission. There was no significant difference in the serum albumin level upon admission between the shallow pressure ulcer (Stage I) group and the deep pressure ulcer (Stages II to IV) group. However, there was a significant difference in the albumin level upon admission between patients in whom the pressure ulcer healed or did not heal during treatment. These results suggest that the serum albumin level is not a predictor of pressure ulcer development but that it may be a good prognostic indicator of wound healing.

To investigate the relation between the serum albumin level and survival, we compared the serum albumin levels between patients who were discharged and those who died during their hospitalization. In our severely ill patients, the serum albumin level was low upon admission and it decreased further as their conditions worsened. Among patients who died in the hospital, the albumin level upon admission and the final level recorded were significantly lower than the corresponding levels in patients who were discharged. These results suggest that the serum albumin level reflects inflammation and disease severity and is closely related to survival outcomes in patients.
Results of our study indicate that assessing nutrition on the basis of serum albumin alone is inadequate because the serum albumin level is not always high in patients with good caloric intake and a low serum albumin level does not always mean malnutrition. We believe the serum albumin level to be a useful indicator of patient prognosis and nutritional risk (risk of malnutrition) as they pertain to the severity of the disease and mortality because the serum albumin level responds well to inflammation, and hypermetabolism is related to the severity of disease including pressure ulcers.

In conclusion, for assessment of nutritional status, we advise clinicians to use not only the serum albumin level but also various indicators such as clinical history, results of physical examination (body weight, amount of muscle and fat), and standard blood test results. Whether the serum albumin level is high or low, patients should be individually provided with adequate nourishment.

\section{CONFLICT OF INTEREST}

None of the authors have any conflicts of interest to declare.

\section{ACKNOWLEDGMENTS}

The study was supported by departmental resources only.

\section{REFERENCES}

1. Butterworth CE Jr: The skeleton in the hospital closet. Nutr Today $9: 4-8,1974$

2. Pinchcofsky GD, Kaminski MV Jr : Increasing malnutrition during hospitalization : documentation by a nutritional screening program. J Am Coll Nutr 4 : 471-479, 1985

3. Tuten MB, Wogt S, Dasse F, Leider Z : Utilization of prealbumin as a nutritional parameter. JPEN J Parenter Enteral Nutr 9 : 709-711, 1985

4. Church JM, Hill GL: Assessing the efficacy of intravenous nutrition in general surgical patients : dynamic nutritional assessment with plasma proteins. JPEN J Parenter Enteral Nutr $11: 135-139,1987$

5. Thorsdottir I, Gunnarsdottir I, Eriksen B : Screening method evaluated by nutritional 
status measurements can be used to detect malnourishment in chronic obstructive pulmonary disease. J Am Diet Assoc 101 : 648-654, 2001

6. Bernstein LH, Leukhardt-Fairfield CJ, Pleban W, Rudolph R: Usefulness of data on albumin and prealbumin concentrations in determining effectiveness of nutritional support. Clin Chem 35 : 271-274, 1989

7. Mowé M, Bøhmer T: The prevalence of undiagnosed protein-calorie undernutrition in a population of hospitalized elderly patients. J Am Ger Soc 39 : 1089-1092, 1991

8. Hanan K, Scheele L : Albumin vs. weight as a predictor of nutritional status and pressure ulcer development. Ostomy Wound Manage 33 : 22-27, 1991

9. Krantz MJ, Lee D, Donahoo WT, Mehler PS : The paradox of normal serum albumin in anorexia nervosa : a case report. Int J Eat Disord 37 : 278-280, 2005

10. Gabay C, Kushner I : Acute-phase proteins and other systemic responses to inflammation. $\mathrm{N}$ Engl J Med 340 : 448-454, 1999

11. Sganga G, Siegel JH, Brown G, Coleman B, Wiles CE 3rd, Belzberg H, Wedel S, Placko R: Reprioritization of hepatic plasma protein release in trauma and sepsis. Arch Surg 120 : 187-199, 1985

12. McClave SA, Mitoraj TE, Thielmeier KA, Greenburg RA : Differentiating subtypes (hypoalbuminemic vs marasmic) of protein-calorie malnutrition : incidence and clinical significance in a university hospital setting. JPEN J Parenter Enteral Nutr $16: 337-342,1992$

13. Kaysen GA, Rathore V, Shearer GC, Depner TA : Mechanisms of hypoalbuminemia in hemodialysis patients. Kidney Int $48: 510-516,1995$

14. Vanek V: The use of serum albumin as a prognostic or nutritional marker and the pros and cons of IV albumin therapy. Nutr Clin Prac 13 : 110-122, 1998

15. Johnson AM : Low levels of plasma proteins : malnutrition or inflammation? Clin Chem Lab Med 37 : 91-96, 1999

16. Fuhrman MP: The albumin-nutrition connection : separating myth from fact. Nutrition 18 : 199-200, 2002

17. Waltman NL, Bergstrom N, Armstrong N, Norvell K, Braden B : Nutritional status, pressure sores, and mortality in elderly patients with cancer. Oncol Nurs Forum 18 : 867-873,
1991

18. Lewicki LJ, Mion L, Splane KG, Samstag D, Secic M : Patient risk factors for pressure ulcers during cardiac surgery. AORN J 65 : 933942, 1997

19. Anthony D, Reynolds T, Russell L : An investigation into the use of serum albumin in pressure sore prediction. J Adv Nurs $32: 359-365$, 2000

20. Gabay C, Kushner I : Acute-phase proteins and other systemic responses to inflammation. $\mathrm{N}$ Engl J Med 340 : 448-454, 1999

21. Andrews NC : Anemia of inflammation : the cytokine-hepcidin link. J Clin Invest 113 : 12511253, 2004

22. Gray GE, Meguid MM : Can total parenteral nutrition reverse hypoalbuminaemia in oncology patients? Nutrition $6: 225-228,1990$

23. Klein $\mathrm{S}$ : The myth of serum albumin as a measure of nutritional status. Gastroenterology 99 : 1845-1846, 1990

24. Mears E : Outcomes of continuous process improvement of a nutritional care program incorporating serum prealbumin measurements. Nutrition $12: 479-484,1996$

25. Seltzer MH, Bastidas JA, Cooper DM, Engler P, Slocum B, Fletcher HS : Instant nutritional assessment. JPEN J Parenter Enteral Nutr 3 : 157-159, 1979

26. Anderson CF, Wochos DN : The utility of serum albumin values in the nutritional assessment of hospitalized patients. Mayo Clin Proc $57: 181-184,1982$

27. Boosalis MG, Ott L, Levine AS, Slag MF, Morley JE, Young B, McClain CJ : Relationship of visceral proteins to nutritional status in chronic and acute stress. Crit Care Med 17 : 741-747, 1989

28. Franch-Arcas $\mathrm{G}$ : The meaning of hypoalbuminaemia in clinical practice. Crit Nutr $20: 265$ 269, 2001

29. Fuhrman MP, Charney P, Mueller CM : Hepatic proteins and nutrition assessment. J Am Diet Assoc 104 : 1258-1264, 2004

30. Myron Johnson A, Merlini G, Sheldon J, Ichihara K : Scientific Division Committee on Plasma Proteins (C-PP), International Federation of Clinical Chemistry and Laboratory Medicine (IFCC). Clinical indications for plasma protein assays : transthyretin (prealbumin) in inflammation and malnutrition. Clin Chem Lab Med 45 : 419-426, 2007 